\title{
Supersymmetry between Jackiw-Nair and Dirac-Majorana anyons
}

\author{
Peter A. Horváthy ${ }^{a}$, Mikhail S. Plyushchay ${ }^{b, c}$, Mauricio Valenzuela $^{a}$ * \\ ${ }^{a}$ Laboratoire de Mathématiques et de Physique Théorique, Université de Tours, \\ Parc de Grandmont, F-37200 Tours, France \\ ${ }^{b}$ Departamento de Física, Universidad de Santiago de Chile, Casilla 30\%, Santiago 2, Chile \\ ${ }^{c}$ Departamento de Física Teórica, Atómica y Óptica, Universidad de Valladolid, 47071, Valladolid, Spain
}

March 7, 2018

\begin{abstract}
The Jackiw-Nair description of anyons combines spin-1 topologically massive fields with the discrete series representation of the Lorentz algebra, which has fractional spin. In the DiracMajorana formulation the spin-1 part is replaced by the spin $1 / 2$ planar Dirac equation. The two models are shown to belong to an $N=1$ supermultiplet, which carries a super-Poincaré symmetry.
\end{abstract}

In the by now standard description of anyons due to Jackiw and Nair [1, the spin 1 representation carried by the topologically massive (TM) vector system [2, 3] is combined with fractional spin. The latter is carried by an internal space (namely the Poincaré disc model of the Lobachevsky plane) [4], described by a complex coordinate $z$. A "Jackiw-Nair" (JN) wave function is,

$$
F_{\mu}(z, x)=\sum_{n} f_{n}(z) F_{\mu}^{n}(x)
$$

where the $f_{n}=c_{n} z^{n}, c_{n}=\sqrt{\Gamma(2 \alpha+n) / \Gamma(2 \alpha) \Gamma(n+1)}$, is restricted to the unit-disk [1, 4]. The $f_{n}, n=0,1,2, \ldots$, span an infinite dimensional orthonormal basis in internal space. $F_{\mu}^{n}(x)$ is, for each internal index $n$, a TM wave function. Then Jackiw and Nair propose to describe anyons by the equations

$$
\left(P^{\mu} \mathfrak{J}_{\mu}^{+}-\beta_{+} m\right) F=0, \quad \beta_{+}=\alpha-1,
$$

where $\alpha>01$. Here $F=\left(F_{\mu}\right)$ and $\mathfrak{J}_{\mu}^{+}$generates the direct sum of Lorentz algebras, $\mathfrak{J}_{\mu}^{+}=J_{\mu}^{+}+j_{\mu}$, where $\left(J_{\mu}^{+}\right)_{\nu}{ }^{\lambda}=i \epsilon_{\mu \nu}{ }^{\lambda}$ generates the spin 1 representation of the Lorentz algebra, and $j_{\mu}$, carrying a fractional spin, belongs to the discrete series of the Lorentz algebra $\left(D_{\alpha}^{+}\right)[1] . J_{\mu}^{+}$acts on the vector index of the field $F_{\mu}$, and $j_{\mu}$ acts on its "fractional" part, labeled by $n$. The "internal" representation can be realized as,

$$
j_{0}=z \partial_{z}+\alpha, \quad j_{1}=-\frac{1+z^{2}}{2} \partial_{z}-\alpha, \quad j_{2}=-i \frac{1-z^{2}}{2} \partial_{z}+i \alpha
$$

\footnotetext{
*e-mails: horvathy-at-univ-tours.fr; mplyushc-at-lauca.usach.cl; valenzuela-at-lmpt.univ-tours.fr

1 The restriction to $\alpha>0$ can in fact be removed, and leads to interpolating anyons which correspond to non-unitary representations [5].
} 
The Lorentz Casimir $j^{\mu} j_{\mu}=-\alpha(\alpha-1)$ is constant, so the representation is irreducible.

Eqn. (2) fixes only one of the Casimirs of the planar Poincaré group, and must therefore be supplemented with subsidiary conditions. Those chosen by Jackiw and Nair [1] are equivalent to

$$
P^{\mu} F_{\mu}=0, \quad \epsilon^{\mu \nu \lambda} P_{\mu} j_{\nu} F_{\lambda}=0 .
$$

Eqns. (2) and (41) imply the Klein-Gordon equation with mass $m$, while (2) fixes the second Casimir operator of the Poincaré group,

$$
P^{\mu}\left(-\epsilon_{\mu \nu \lambda} x^{\nu} P^{\lambda}+\mathfrak{J}_{\mu}^{+}\right)=\beta_{+} m .
$$

Hence, the spin is $\beta_{+}=\alpha-1$. Eqns. (2) and (4) imply the equation of the TM theory,

$$
\mathfrak{D}_{\mu}{ }^{\nu} F_{\nu} \equiv\left(-i \epsilon_{\mu \lambda}{ }^{\nu} P^{\lambda}+m \delta_{\mu}^{\nu}\right) F_{\nu}=0
$$

Conversely, it can be shown [5] that (22) and (44) together are equivalent to imposing the TM equations (6), augmented with the Majorana equation

$$
\left(P^{\mu} j_{\mu}-\alpha m\right) F=0 .
$$

The Jackiw-Nair theory is, hence, equivalent to the coupled TM-Majorana system (6)-(7).

In another, slightly different approach [6], the anyon field is described rather by a spinor,

$$
\psi_{a}(x, z)=\sum_{n} g_{n}(z) \psi_{a}^{n}(x), \quad\left(P^{\mu} \mathfrak{J}_{\mu}^{-}-\beta_{-} m\right) \psi=0, \quad \beta_{-}=\alpha-\frac{1}{2}
$$

where the $\mathfrak{J}_{\mu}^{-}=J_{\mu}^{-}+j_{\mu}$ with $\left(J_{\mu}^{-}\right)_{a}{ }^{b}=-\frac{1}{2}\left(\gamma_{\mu}\right)_{a}{ }^{b}$ generate the spin $1 / 2$ representation of the planar Lorentz group. Instead of the TM equation (6), the field is required to satisfy the planar Dirac equation,

$$
\mathcal{D}_{a}^{b} \psi_{b} \equiv\left(P_{\mu} \gamma^{\mu}-m\right)_{a}^{b} \psi_{b}=0 .
$$

Note that the Dirac (9) and Majorana (77) equations [with $\psi$ replacing $F$ ] imply

$$
\left(j_{\mu} \gamma^{\mu}+\beta_{-}\right) \psi=0, \quad i \epsilon^{\mu \nu \lambda} P_{\mu} j_{\nu} \gamma_{\lambda} \psi=0
$$

as consistency conditions, which eliminate the redundant modes [6].

The aim of this Note is to show that the Jackiw-Nair and Dirac-Majorana approaches are two facets of the same supersymmetric system : they are in fact, superpartners. To this end, we note first that a fractional spin field can be described, in both approaches, by equations of the same form,

$$
D^{ \pm} \psi^{ \pm}=0, \quad\left(P^{\mu} j_{\mu}-\alpha m\right) \psi^{ \pm}=0, \quad \text { with spin }\left\{\begin{array}{l}
\beta_{-}=\alpha-\frac{1}{2} \\
\beta_{+}=\alpha-1
\end{array}\right.
$$

where $D^{+}=\mathfrak{D}$ and $D^{-}=\mathcal{D}$ are the operators in TM and the Dirac equations, (6) and (9), respectively, and we put $\psi^{-}=\psi$ and $\psi^{+}=F$. We note for further reference that, in both frameworks, the posited first-order equations imply that the field satisfies the Klein-Gordon equation. 
The (fractional) spins of the fields $\psi^{-}$and $\psi^{+}$are shifted by $\beta_{-}-\beta_{+}=1 / 2$, and have the same masses. They can therefore be unified into a supermultiplet along the same lines as done recently for the TM and Dirac fields [5, 7]. We posit

$$
\left(P_{\mu} \mathcal{J}^{\mu}-\hat{\alpha} m\right) \Psi=0, \quad\left(P^{\mu} j_{\mu}-\alpha m\right) \Psi=0
$$

where $\Psi$ is formed by putting together the Dirac-Majorana and Jackiw-Nair (11) fields, $\Psi=$ $\left(\begin{array}{l}\psi^{-} \\ \psi^{+}\end{array}\right)$, and $\hat{\alpha}$ is $\alpha_{-}=-\frac{1}{2}$ for $\psi=\left(\begin{array}{l}\psi^{-} \\ 0\end{array}\right)$, and $\alpha_{+}=-1$, for $\psi=\left(\begin{array}{l}0 \\ \psi^{+}\end{array}\right)$. The first equation in (12) is the supersymmetric equation which unifies the Dirac and TM equations [7], and is supplemented by the Majorana equation.

The total spin operator, $\hat{\beta}=\hat{\alpha}+\alpha=\operatorname{diag}\left(\beta_{-} \mathbb{1}_{2}, \beta_{+} \mathbb{1}_{3}\right)$, takes the value $\beta_{ \pm}$on the subspaces spanned by the Dirac-Majorana and the Jackiw-Nair field, respectively.

Lorentz transformations are generated by $\mathfrak{M}_{\mu}=-\epsilon_{\mu \nu \lambda} x^{\nu} P^{\lambda}+\mathfrak{J}_{\mu}$ where $\mathfrak{J}_{\mu}=\operatorname{diag}\left(\mathfrak{J}_{\mu}^{-}, \mathfrak{J}_{\mu}^{+}\right)$ is block-diagonal with irreducible components acting on the Dirac-Majorana and Jackiw-Nair components of the supermultiplet. Augmented with translations, $P_{\mu}$, yields the Poincaré algebra,

$$
\left[P_{\mu}, P_{\nu}\right]=0, \quad\left[\mathfrak{M}_{\mu}, P_{\nu}\right]=-i \epsilon_{\mu \nu \lambda} P^{\lambda}, \quad\left[\mathfrak{M}_{\mu}, \mathfrak{M}_{\nu}\right]=-i \epsilon_{\mu \nu \lambda} \mathfrak{M}^{\lambda}
$$

The Lorentz algebra generated by $\mathcal{J}_{\mu}$ can be extended into the superalgebra $\mathfrak{o s p}(1 \mid 2)$ by adding the off-diagonal matrices 2

$$
L_{\underline{A}}=\sqrt{2}\left(\begin{array}{cc}
0 & Q_{\underline{A}} a^{\mu} \\
Q_{\underline{A}}{ }^{a} & 0
\end{array}\right), \quad Q_{\underline{1}} a^{\mu}=\left(\begin{array}{ccc}
0 & 1 & i \\
1 & 0 & 0
\end{array}\right), \quad Q_{\underline{\underline{2}} a}{ }^{\mu}=\left(\begin{array}{ccc}
1 & 0 & 0 \\
0 & 1 & -i
\end{array}\right) .
$$

The operators $L_{\underline{A}}$ form a Lorentz spinor, $\left[\mathfrak{J}_{\mu}, L_{\underline{A}}\right]=\frac{1}{2}\left(\gamma_{\mu}\right)_{\underline{A}}^{\underline{B}} L_{\underline{B}}$ and interchange $\psi$ and $F$ (where we returned to our original notations).

They do not preserve the physical states defined as solutions of the Dirac and TM equations, respectively. Consider instead the supercharges

$$
\mathcal{Q}_{\underline{A}}=\frac{1}{2 \sqrt{m}}\left(P_{\mu} \gamma^{\mu}-R m\right)_{\underline{A}}^{\underline{B}} L_{\underline{B}}
$$

where $R=\operatorname{diag}\left(-\mathbb{1}_{2}, \mathbb{1}_{3}\right)$ is the reflection operator, $\left\{R, L_{\underline{A}}\right\}=0$, which transform a twocomponent Dirac field into a three-component TM field $F^{\prime}$ and conversely. Explicitly,

$$
\mathcal{Q}_{\underline{A}} \Psi=\left(\begin{array}{c}
\psi_{a}^{\prime} \\
F_{\mu}^{\prime}
\end{array}\right)=\left(\begin{array}{c}
\sum_{n} f_{n}(z) \mathcal{Q}_{\underline{A}} a^{\mu} F_{\mu}^{n}(x) \\
\sum_{n} g_{n}(z) \mathcal{Q}_{\underline{A} \mu}{ }^{a} \psi_{a}^{n}(x)
\end{array}\right) .
$$

A general SUSY transformation is a linear combination of the $\mathcal{Q}_{\underline{A}} \mathrm{~s}, \mathcal{Q}=\zeta^{\underline{A}} \mathcal{Q}_{\underline{A}}$. Moreover,

$$
\begin{aligned}
& \mathcal{D}_{a}{ }^{b} \psi_{b}^{\prime}=\zeta^{\underline{A}}\left(\mathcal{Q}_{\underline{A} a}{ }^{\mu} \mathfrak{D}_{\mu}{ }^{\nu} F_{\nu}+\frac{1}{2 \sqrt{m}} Q_{\underline{A}} a^{\mu}\left(P^{2}+m^{2}\right) F_{\mu}\right), \\
& \mathfrak{D}_{\mu}{ }^{\nu} F_{\nu}^{\prime}=\zeta^{\underline{A}}\left(-\frac{1}{2} \mathcal{Q}_{\underline{A} \mu}{ }^{a} \mathcal{D}_{a}{ }^{b} \psi_{b}-\frac{1}{2 \sqrt{m}} Q_{\underline{A} \mu}{ }^{a}\left(P^{2}+m^{2}\right) \psi_{a}\right),
\end{aligned}
$$

showing that $\psi_{b}^{\prime}$ satisfies the Dirac equation if and only if $F_{\nu}^{\prime}$ satisfies the TM equation.

\footnotetext{
${ }^{2}$ Underlined capitals denote $\mathfrak{o s p}(1 \mid 2)$ spinors.
} 
Now the Majorana equations are intertwined by the SUSY transformation,

$$
\begin{aligned}
& \left(P^{\mu} j_{\mu}-\alpha m\right) \psi_{a}^{\prime}=\zeta^{\underline{A}}\left(\mathcal{Q}_{\underline{A} a}{ }^{\mu}\left(P^{\nu} j_{\nu}-\alpha m\right) F_{\mu}\right)=0, \\
& \left(P^{\nu} j_{\nu}-\alpha m\right) F_{\mu}^{\prime}=\zeta^{\underline{A}}\left(\mathcal{Q}_{\underline{A} \mu}{ }^{a}\left(P^{\nu} j_{\nu}-\alpha m\right) \psi_{a}\right)=0,
\end{aligned}
$$

allowing us to conclude that the $\mathcal{Q}_{\underline{A}}$ in (15) generate indeed a supersymmetry transformation betwen the two, DM and JN, sectors.

Completing the Poincaré algebra (13) by the supercharges (15),

$$
\begin{gathered}
{\left[P_{\mu}, \mathcal{Q}_{\underline{A}}\right]=0, \quad\left[\mathfrak{M}_{\mu}, \mathcal{Q}_{\underline{A}}\right]=-\frac{1}{2}\left(\gamma_{\mu}\right)_{\underline{A}}^{\underline{B}} \mathcal{Q}_{\underline{B}},} \\
\left\{\mathcal{Q}_{\underline{A}}, \mathcal{Q}_{\underline{B}}\right\}=2(P \gamma)_{\underline{A B}}+\frac{1}{2 m}\left[(\mathcal{J} \gamma)_{\underline{A B}}\left(P^{2}+m^{2}\right)-2(P \gamma)_{\underline{A B}}(P \mathcal{J}-\hat{\alpha} m)\right],
\end{gathered}
$$

where $(P \gamma)_{\underline{A B}}$ means $P^{\mu} \gamma_{\mu_{\underline{A}}} \epsilon_{\underline{C B}}$.

On shell, the unified system carries therefore an $N=1$ superPoincaré symmetry. The superCasimir is $C=P^{\mu} \mathcal{J}_{\mu}-\frac{1}{16}\left[\mathcal{Q}_{\underline{1}}, \mathcal{Q}_{2}\right]=m(\alpha-3 / 4)$, a constant, showing that the representation is indeed irreducible.

We note, in conclusion, that the supersymmetry of the two, Dirac-Majorana (DM) and JackiwNair (JN) types of anyons proved here explicitly had to be expected from that of the "carrying" spin $1 / 2$ and spin 1 spaces [2, 5].

Acknowledgements. Partial support by the FONDECYT (Chile) under the grant 1095027 and by DICYT (USACH), and by Spanish Ministerio de Educación under Project SAB2009-0181 (sabbatical grant of MSP), is acknowledged. MV has been supported by CNRS postdoctoral grant (contract number 87366).

\section{References}

[1] R. Jackiw and V. P. Nair, Phys. Rev. D 43 (1991) 1933.

[2] J. F. Schonfeld, Nucl. Phys. B 185 (1981) 157.

[3] S. Deser, R. Jackiw and S. Templeton, Annals Phys. 140 (1982) 372; Phys. Rev. Lett. 48 (1982) 975.

[4] M. S. Plyushchay, Nucl. Phys. B 362 (1991) 54.

[5] P. A. Horvathy, M. S. Plyushchay and M. Valenzuela, Annals of Physics (2010), doi: 10.1016/j.aop.2010.02.007 [arXiv: 1001.0274].

[6] M. S. Plyushchay, Phys. Lett. B 273 (1991) 250.

[7] P. A. Horvathy, M. S. Plyushchay and M. Valenzuela, [arXiv: 1002.4729]. 